\title{
Reactivation of Sarin-inhibited Pig Brain Acetylcholinesterase using Oxime Antidotes
}

\author{
Kamil Kuca, PhD, and Daniel Jun, PhD
}

Centre of Advanced Studies, Department of Toxicology, Faculty Military Health Sciences, University of Defence, Hradec Kralove, Czech Republic

\begin{abstract}
Introduction: Organophosphorus nerve agents inhibit the enzyme, acetylcholinesterase (AChE; EC 3.1.1.7). AChE reactivators (also known as oximes) are generally used for the reactivation of an inhibited enzyme.

Methods: Two new AChE reactivators-K033 and K027-were tested for their in vitro reactivation of sarin-inhibited pig-brain AChE. Their reactivation potencies were compared with the commercially available AChE reactivators, pralidoxime, obidoxime, and HI-6.

Results: Of the oximes tested, the newly developed oxime K027 achieved the highest reactivation potency (100\%; concentration of the oxime $-10^{-2} \mathrm{M}$ ). However, oxime HI-6 (33\%) and obidoxime (23\%) seem to be the best AChE reactivators for human relevant doses $\left(10^{-4} \mathrm{M}\right.$ and lower).

Conclusion: For human relevant doses, newly developed oximes (K027 and K033) do not surpass the reactivation potency of the most promising oxime, HI-6.
\end{abstract}

\section{INTRODUCTION}

Organophosphorus compounds (OPCs) are utilized in industry as softening agents, hydraulic liquids, lubricant additives, plasticizers, antioxidants, and antiflammable modifications. They are also used in animals and humans as drugs or as chemicals for the study of nervous function. Unfortunately, these compounds are also used for military purposes as chemical warfare agents (nerve agents) and as poisons exploited by terrorists [1].

The toxicity of OPCs, especially nerve agents, is principally due to their ability to inhibit the enzyme acetylcholinesterase (AChE; EC 3.1.1.7) by phosphorylation or phosphonylation of the serine hydroxy group in its active site $[2,3]$. Owing to the fact that the time required for spontaneous reactivation is comparable to the natural lifetime of AChE in the body, the inhibition is designed to be irreversible. After the inhibition process, the enzyme is not able to serve its mission to cleave neuromediator acetylcholine (ACh). The resulting accumulation of ACh at synaptic junctions over-stimulates cholinergic pathways and subsequently desensitizes cholinergic receptor sites $[1,2,4,10]$.

Standard treatment of toxicity consists of a combination of anticholinergic drugs to counteract the effect of accumulated ACh (e.g. atropine) and AChE reactivators to reactivate OPCsinhibited AChE. Monoquaternary pralidoxime (2-PAM), bisquaternary obidoxime, and oxime HI-6 are typical members of the family of AChE reactivators (Figure 1) [5].

AChE reactivators, also known as oximes, are able to displace the organosphosphorus moiety from the inhibited

Keywords: acetylcholinesterase, reactivation, sarin, nerve agents, inhibition, pig brain homogenate

Acknowledgements: The authors would like to thank to Mrs. Martina Hrabinova and Mr. Petr Stodulka for their technical assistance. This work was supported by a grant from the Ministry of Education, Youth and Sports (Czech Republic) No. ME865.

Notes: There was no outside funding of any kind used for this study.

Corresponding Author: Kamil KUCA, PhD, Department of Toxicology, Faculty of Military Health Sciences, University of Defence, Trebesska 1575. 500 01, Hradec Kralove, Czech Republic. Email: kucakam@pmfhk.cz 


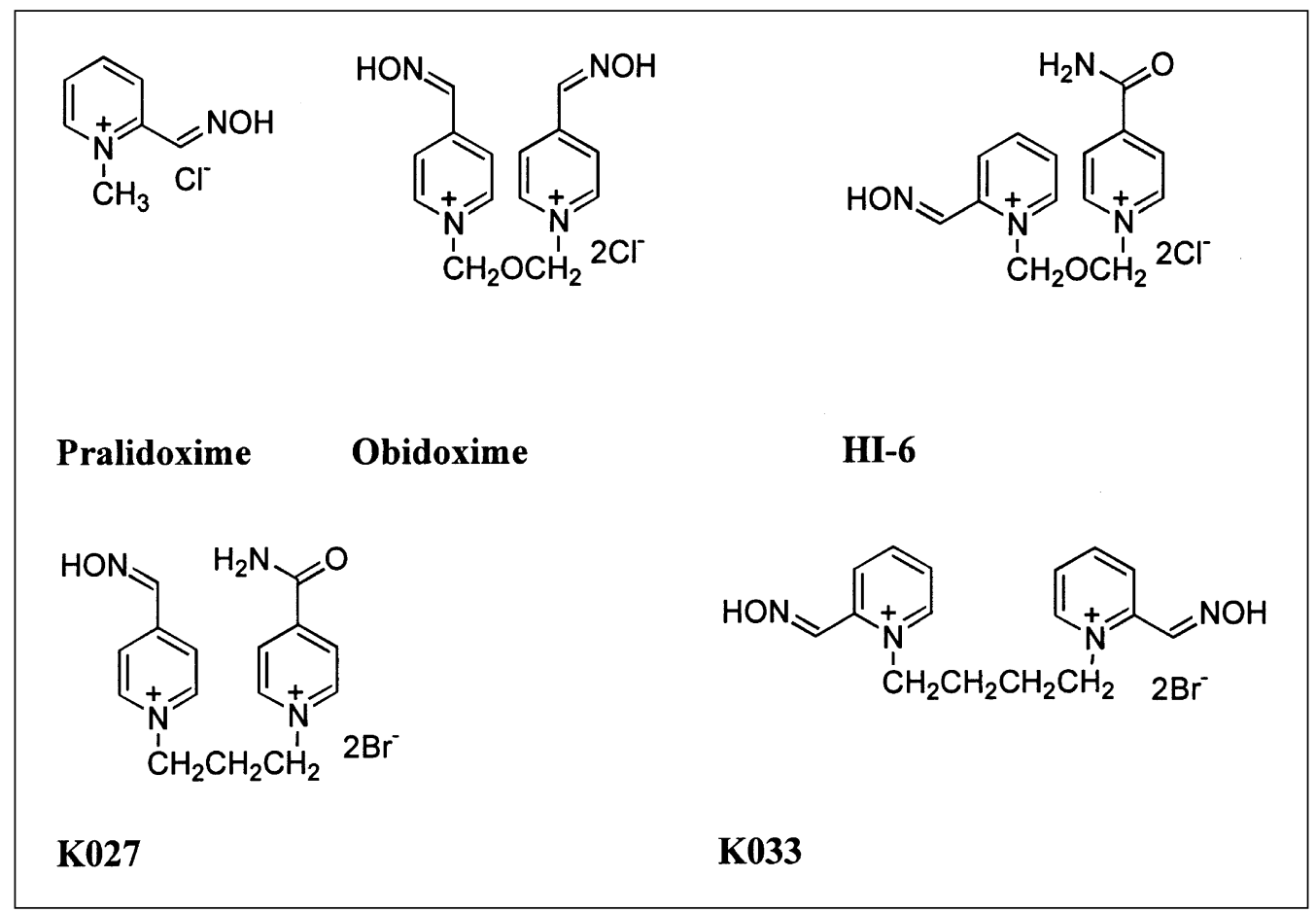

Figure 1. Structure of Currently used oximes and their new analogues.

enzyme. An oxime group in human blood dissociates, and the remaining oximate anion acts as a nucleophile and cleaves the bond between the enzyme and the phosphorus atom of the inhibitor. A regenerated, functional enzyme is the result of the reactivation process.

The above mentioned oximes are effective against most of the OPCs, but their therapeutic effectiveness is not completely satisfactory against other OPCs, especially nerve agents. For example, pralidoxime is unable to reactivate tabun-inhibited and cyclosarin-inhibited AChE in vitro and in vivo [6-8]. Similar results were obtained for obidoxime. Obidoxime has no ability to reactivate cyclosarin-inhibited AChE in vitro, and its potency to reactivate tabun-inhibited AChE in vitro is poor [9]. Currently the most promising AChE reactivator, oxime HI-6, is the strongest in vitro and in vivo reactivator against almost all of the nerve agents $[5,10]$. Unfortunately, it has no potency to reactivate tabuninhibited AChE [6,7]. Therefore, the replacement of currently used AChE reactivators with new and more effective oximes is a long-standing goal for the treatment of poisonings associated with highly toxic OPCs.

During the last several years, we have synthesized two new AChE reactivators (K033 and K027) at our department (Figure 2) $[11,12]$. Based on their potencies in reactivating rat brain AChE inhibited by several nerve agents (sarin, tabun, VX and cyclosarin) in vitro, they would be expected to be promising AChE reactivators $[13,14]$.

Unfortunately, all in vitro reactivation tests were only done with the rat-brain homogenate. Although in vitro measurements with rodents are always needed because of subsequent in vivo testing with the same animal, results with another animal species are necessary. Previous studies indicated that the ability of oximes to reactivate AChE differed between species [15].

In order to obtain some new information about the potency of the new AChE reactivators (K033 and K027), we tested their abilities to reactivate pig-brain AChE inhibited by sarin. Their reactivation potencies were compared with three commonly used AChE reactivators, pralidoxime, obidoxime, and HI-6. Later, we discuss the relationship between the structure of AChE reactivators and their biological affinity.

\section{MATERIAL AND METHODS}

\section{Chemicals}

AChE reactivators (pralidoxime and obidoxime) were purchased from Leciva (Czech Republic) and Merck (Germany), respectively. HI-6 and the new oximes, K033 and K027, were synthesized at our department $[11,12,13,16]$. The purity of all AChE reactivators was tested using TLC (DC-Alufolien Cellulose F; mobile phase n-butanol: acetic acid: water-5:1:2; Detection: Dragendorff reagent).

Sarin (GB; O-isopropyl methylflourophosphonate) was obtained from the Military Technical Institute (Brno, Czech Republic) and was 95\% pure. The purity was evaluated by acidimetric titration. 


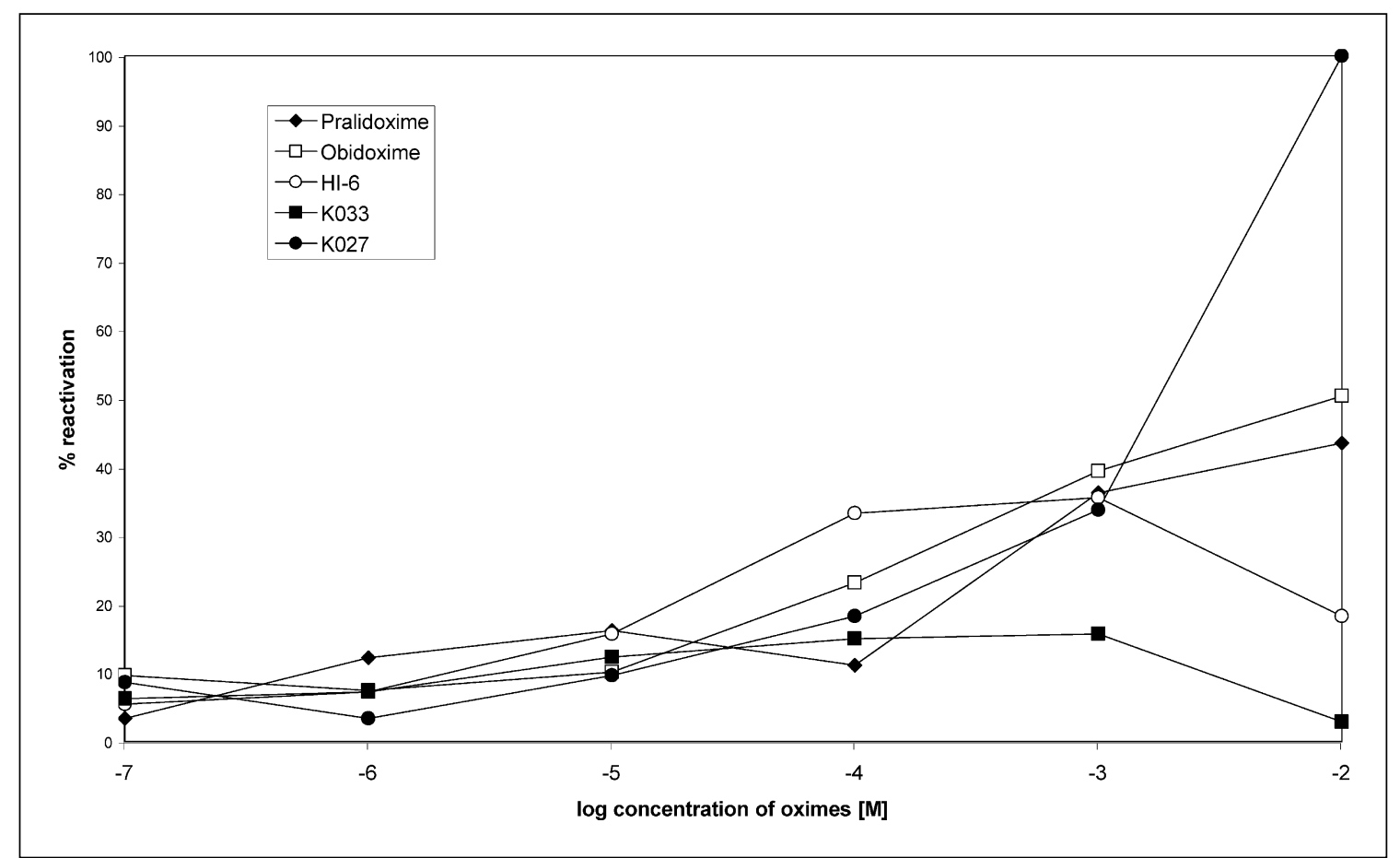

Figure 2. Reactivation-concentration relationships of oximes to sarin-inhibited AChE (source of enzyme-pig brain homogenate; nerve agent-sarin; time of inhibition by nerve agent-30 min; time of reactivation by oxime-10 min; temperature $25^{\circ} \mathrm{C}$; $\mathrm{pH} 7,6$; $n=3)$

\section{Enzyme}

Pig brain homogenate was used as the appropriate source of the enzyme (AChE). The euthanasia of animals was provided by intravenous administration of embutramid in combination with mebezonium iodide and tetracaine hydrochloride (T61, Intervet International B.V., Netherlands). The whole brain was excised, washed with saline, and homogenized with distilled water $(10 \%$ $\mathrm{w} / \mathrm{v}$ ) by the use of an Ultra-Turrax instrument (Janke-Kunkel, Germany) at $20.000 \mathrm{rpms}$ for $1 \mathrm{~min}$. Aliquots $(2 \mathrm{ml})$ of the homogenate were stored at $-25^{\circ} \mathrm{C}$ in a freezer and were thawed immediately prior to use.

\section{In vitro experiments}

All tested AChE reactivators were tested using a standard reactivation test described earlier by Kuca and Kassa [14].

Percentage of the reactivation at specific concentration was calculated from the equation: $\% \mathrm{R}=\left[1-\left(\mathrm{a}_{0}-\mathrm{a}_{\mathrm{R}}\right) /\left(\mathrm{a}_{0}-\mathrm{a}_{\mathrm{T}}\right)\right] \times 100$, where $\left(\mathrm{a}_{0}\right)$ is activity of the intact enzyme, $\left(\mathrm{a}_{\mathrm{I}}\right)$ is activity of inhibited enzyme, and $\left(a_{R}\right)$ is activity of reactivated enzyme.

Reactivation potency of the tested oximes is characterized by several constants. Dissociation constant $K_{\mathrm{R}}$ describes a creation of the complex of the reactivator with the inhibited enzyme. Compounds with lower values of this constant have higher affinity to the inhibited enzyme. Rate constants $k_{\mathrm{R}}$ and $k_{\mathrm{r}}$ describe a velocity of the reactivation of inhibited AChE. The first order rate constant $k_{\mathrm{R}}$ characterizes the creation of the inhibited enzyme- reactivator complex. Second order rate constant $k_{\mathrm{r}}$ characterizes the velocity of the overall reaction, and it is derived from the equation $k_{\mathrm{r}}=k_{\mathrm{R}} / K_{\mathrm{R}}[16]$.

A dissociation constant of the intact enzyme-reactivator $K_{\text {DIS }}$ was obtained by a non-linear regression from the dependence of $a_{\mathrm{I}}$ on $C_{\mathrm{OX}}$ by using the following equation: $\mathrm{a}_{\mathrm{I}}=\mathrm{a}_{\max }$ $\mathrm{C}_{\mathrm{S}} /\left[\mathrm{C}_{\mathrm{S}}+\mathrm{K}_{\mathrm{M}}\left(1+\mathrm{C}_{\mathrm{OX}} / \mathrm{K}_{\mathrm{DIS}}\right)\right]$, where, $\mathrm{a}_{\max }$ denotes the maximum (limiting) rate of the enzymatic reaction; $C_{\mathrm{S}}$ is the substrate concentration; $K_{\mathrm{M}}$ is the Michaelis constant for the hydrolysis of acetylcholine with AChE $\left(1.9 \times 10^{-4} \mathrm{~mol} \mathrm{l}^{-1}\right)$; and $C_{\mathrm{OX}}$ is an appropriate concentration of the tested oxime.

\section{RESULTS}

The values of all constants written in Figure 3 describe the whole reactivation process. Oxime $\mathrm{K} 033$ has the highest affinity towards the intact enzyme. Its value is from 4 to 6 times lower compared to HI-6, obidoxime, and K027. Pralidoxime has the highest value of the dissociation constant $K_{\mathrm{DIS}}$, the lowest affinity towards the intact enzyme.

Similar results were obtained for the dissociation constant $K_{\mathrm{R}}$, which describes affinity of the AChE reactivator towards the inhibited enzyme. Oxime HI- 6 has the highest affinity. Similar values were obtained for obidoxime, K027, and K033. Pralidoxime achieved the lowest affinity towards the inhibited enzyme too. 


\begin{tabular}{|c|c|c|c|c|}
\hline Oxime & $\boldsymbol{K}_{\mathrm{DIS}}[\mu \mathbf{M}]$ & $\boldsymbol{K}_{\mathbf{R}}[\mu \mathbf{M}]$ & $k_{\mathrm{R}}\left[\min ^{-1}\right]$ & $k_{r}\left[\min ^{-1} \cdot \mu M^{-1}\right]$ \\
\hline Pralidoxime & 1000 & 4074 & 0.230 & 56 \\
\hline Obidoxime & 20 & 355 & 0.100 & 282 \\
\hline $\mathrm{HI}-6$ & 17 & 98 & 0.080 & 816 \\
\hline K027 & 23 & 417 & 0.080 & 192 \\
\hline K033 & 4 & 380 & 0.080 & 211 \\
\hline \multicolumn{5}{|c|}{$\begin{array}{l}K_{\mathrm{D} \mathrm{DI}^{\prime}} \text { dissociation constant of the enzyme-reactivator complex; } K_{\mathrm{R}^{\prime}} \text { dissocia- } \\
\text { tion constant of inhibited enzyme-reactivator complex; } k_{\mathrm{R}^{\prime}} \text { the first-order rate } \\
\text { constant of reactivation; } k_{\mathrm{r}^{\prime}} \text { the second order rate constant of reactivation }\end{array}$} \\
\hline
\end{tabular}

Figure 3. Kinetic constants of the reactivation.

The first order rate constant $k_{\mathrm{R}}$, characterizing the decomposition of the inhibited enzyme-reactivator complex, favors pralidoxime. However, our results of the second order rate constant $k_{\mathrm{r}^{\prime}}$ characterizing the velocity of the whole reactivation process, shows that the most potent reactivator of sarin-inhibited AChE is oxime HI-6. The remaining values decrease as follows: obidoxime $>$ K033 > K027 > pralidoxime.

Visualization of the relationship between the concentration of AChE reactivators and their ability to reactivate sarin-inhibited AChE is shown in Figure 3. According to the obtained results, oxime $\mathrm{K} 027$ seems to be the best AChE reactivator at the concentration $10^{-2} \mathrm{M}$. However, this concentration level could be toxic to humans [17]. Quite different results were obtained at the lower affinities for human relevant concentrations $\left(10^{-4} \mathrm{M}\right.$ and lower) of oximes. At the concentration $10^{-4} \mathrm{M}$, HI- 6 achieved the highest reactivation potency (33\%) among all tested oximes. Reactivation potency of all other oximes tested at this concentration is as follows: obidoxime (23\%), K027 (18\%), K033 (15\%) and pralidoxime (11\%). All tested AChE reactivators are practically ineffective at the low concentrations (in the range from $10^{-5}$ to $10^{-7} \mathrm{M}$ ). Their reactivation potencies are almost similar and their values do not achieve the magnitude needed for a successful reactivation process (10\%) [1].

\section{DISCUSSION}

Current development of prophylaxis and treatment of nerve agent toxicity is focused on scavenger cholinesterases $[1,17,18]$. Unfortunately, this approach only protects against a few $\mathrm{LD}_{50}$ nerve agents [19]. If the amount of nerve agents exceeds the tolerable dose, AChE reactivators (as the causal antidotes) are still required, and so the development of new and more potent $\mathrm{AChE}$ reactivators continues [20-23].

In this study, we have tested three currently used AChE reactivators (pralidoxime, obidoxime, and HI-6) in comparison with our newly developed AChE reactivators (K033 and K027) [11,12]. We have previously tested their potencies on rat brain homogenate $[13,14]$. The use of different animal species makes the effects of AChE reactivators in humans more predictable $[15,25]$.

Of the reactivators tested, HI-6 is the safest AChE reactivator for use in humans. This result supports outcomes obtained from the inhibition of AChE by sarin tested on rat brain homogeate [26]. The newly synthesized oximes are very good reactivators of AChE inhibited by sarin at high concentrations. Unfortunately, they are not able to reactivate sarin-inhibited AChE at low concentrations in the range from $10^{-4}$ to $10^{-7} \mathrm{M}$.

The chemical structure of AChE reactivators affects their ability to reactivate AChE inhibited by nerve agents $[6,8]$. Some of the most important moieties include the presence of the oxime group, the presence of quaternary nitrogen, and the appropriate length of the connection chain between both quaternary nitrogens [27]. All tested AChE reactivators contain the necessary structural factors.

From the substances tested, pralidoxime is the only monoquaternary reactivator nerve agent inhibited by AChE. In the molecule of pralidoxime, only one quaternary nitrogen is incorporated, and its affinity towards an intact and inhibited enzyme is too low. Compared to pralidoxime, all other tested bisquaternary AChE reactivators have a higher affinity towards both kinds of AChE. A satisfactory reactivation process depends on the nucleophile used. Currently, oximate anion is the most suitable nuclephilic group. There are many research studies dealing with the quantitative structure activity relationship (QSAR) [28-30]. QSAR uses structural patterns (position and number of oxime groups, presence and number of quaternary nitrogen) and physico-chemical properties (partition coefficient, $\mathrm{p} K_{\mathrm{a}}$ ) for predicting the biological activity of a proposed AChE reactivator [31]. However, none of them are able to accurately predict the most active, desired structure. This is the reason the in vitro method remains the main technique for quick and cheap evaluation of new AChE reactivators.

\section{CONCLUSION}

Using our in vitro method, we have evaluated the potency of two newly developed AChE reactivators (K033 and K027) and compared them to currently used AChE reactivators. Pig brain homogenate was used as a suitable source of the enzyme. According to our results, if compared with our previous data, the reactivation process is species dependent. Because the reactivation process is species dependent, it should be taken into account that results obtained for one animal species will not be achieved for other animal species (species limitation). Another limitation is in vitro versus in vivo testing. This limitation can be solved by repetition of these experiments in vivo on different animal species. These experiments on larger animals are expensive, and only a few laboratories throughout the world are equipped and interested in the experiments. Moreover, such in vivo experiments on humans are not approved for ethical reasons. For all these reasons, comparative in vitro studies are still needed.

The authors have no potential conflicts of interest to report. 


\section{REFERENCES}

1. Bajgar J. Organophosphates/nerve agent poisoning: mechanism of action, diagnosis, prophylaxis, and treatment. Adv Clin Chem 2004;38,151-216.

2. Marrs TC. Organophosphate poisoning. Pharmacol Therap 1993;58:51-66.

3. Segura-Aguilar J, Kostrzewa RM. Neurotoxins and neurotoxic species implicated in neurodegeneration. Neurotox Res 2004; 6 :615-30.

4. Kassa J. The influence of oxime and anticholinergic drug selectioin on the potency of antidotal treatment to counteract acute toxic effects of tabun in mice. Neurotox Res 2005; (In Press)

5. Kassa J. Review of oximes in the antidotal treatment of poisoning by organophosphorus nerve agents. J Toxicol Clin Toxicol 2002;40:803-16.

6. Cabal J, Kuca K, Kassa J Specification of the structure of oximes able to reactivate tabun inhibited acetylcholinesterase. Bas Clin Pharmacol Toxicol 2004;95:81-6.

7. Kuca K, Cabal J. In vitro reactivation of tabun-inhibited acetylcholinesterase using new oximes-K027, K005, K033 a K048. Centr Eur J Publ Health 2004; 12(Suppl.): S59-S61.

8. Kuca K, Patocka J. Reactivation of cyclosarin-inhibited rat brain acetylcholinesterase by pyridinium-oximes. J Enzyme Inhib Med Chem 2004; 19:39-43.

9. Kassa J, Cabal J. A comparison of the efficacy of acetylcholinesterase reactivators against cyclohexyl methylphosphonofluoridate (GF Agent) by in vitro and in vivo methods. Pharm Toxicol 1999;84:41-5.

10. Sevelova L, Kuca K, Krejcova G. Antidotal treatment of GF-agent intoxication in mice with new bispyridinium oximes. Toxicology 2005;207:1-6.

11. Kuča K., Bielavský J., Cabal J., Bielavská M.: Synthesis of a potential reactivator of acetylcholinesterase 1-(4-hydroxyiminomethylpyridinium)-3-(carbamoylpyridinium)propane dibromide. Tetrahedron Letters 2003;44:3123-3125.

12. Kuca K, Cabal J, Patocka J, Kassa J. Synthesis of bisquaternary symmetric- $\chi, \delta$-bis(2-hydroxyiminomethylpyridinium) alkane dibromides and their reactivation of cyclosarin-inhibited acetylcholinesterase. Lett Org Chem 2004;1:84-6.

13. Kuca K, Cabal J, Kassa J. A comparison of the efficacy of a bispyridinium oxime-1,4-bis-(2hydroxyiminomethylpyridinium) butane dibromide and currently used oximes to reactivate sarin, tabun or cyclosarin-inhibited acetylcholinesterase by in vitro methods. Die Pharmazie 2004;59:795-8.

14. Kuca K, Kassa J. In vitro reactivation of acetylcholinesterase using of the oxime K027. Vet Hum Toxicol 2004; $46: 15-8$.

15. Worek F, Reiter G, Eyer P, Szinicz L. Reactivation kinetics of acetylcholinesterase from different species inhibited by highly toxic organophosphates Arch Toxicol 2002;76:523-9.

16. Patocka J, Bielavsky J, Ornst F. Reactivating effect of alpha, omega-bis(4-pyridinealdoxime)-2-trans-butene dibromide on isopropyl-methylphosphonylated acetylcholinesterase. FEBS Lett 1970;10:182-4.

17. Tattersall JEH. Ion channel blockade by oximes and recovery of diaphragm muscle from soman poisoning in vitro. Br J Pharmacol 1993;108:106-15.

18. Sevelova L, Bajgar J, Saxena A, Doctor BP. Protective effect of equine butyrylcholinesterase in inhalation intoxication of rats with sarin: determination of blood and brain cholinesterase activities. Inhal Toxicol 2004; $16: 531-6$.

19. Broomfield CA, Maxwell DM, Solana RP, Castro CA, Finger AV, Lenz DE. Protection by butyrylcholinesterase against organophosphorus poisoning in nonhuman primates.

J Pharmacol Exp Ther 1991;259:633-8.

20. Bedford CD, Miura M, Bottaro JC, Howd RA, Nolen HW. Nonquaternary cholinesterase reactivators. 4. Dialkylaminoalkyl thioesters of alpha-keto thiohydroximic acids as reactivators of ethyl methylphosphonyl- and 1,2,2-trimethylpropyl methylphosphonyl-acetylcholinesterase in vitro. J Med Chem 1986;29:1689-96.

21. Degorre F, Kiffer D, Terrier F. Sulfur derivatives of 2-oxopropanal oxime as reactivators of organophosphateinhibited acetylcholinesterase in vitro: synthesis and structurereactivity relationships. J Med Chem 1988;31:757-63.

22. Hammond PI, Kern C, Hong F, Kollmeyer TM, Pang YP, Brimijoin S. Cholinesterase reactivation in vivo with a novel bis-oxime optimized by computer-aided design J Pharmacol Exp Therap 2003;307:190-6.

23. Yang GY, Yoon JH, Seong CM, Park NS, Jung YS. Synthesis of Bis-pyridinium oxime antidotes using bis(methylsulfonoxymethyl) ether for organophosphate nerve agents. Bull Korean Chem Soc 2003;24,1368-70.

24. Kuca K, Kassa J. A Comparison of the ability of a new bispyridinium oxime-1-(4-hydroxyiminomethylpyridinium)-4(4-carbamoylpyridinium)butane dibromide and currently used oximes to reactivate nerve agent-inhibited rat brain acetylcholinesterase by in vitro methods. J Enzyme Inhib Med Chem 2003; 18:529-35.

25. Clement JG, Erhardt N. In vitro oxime-induced reactivation of various molecular forms of soman-inhibited acetylcholinesterase in striated muscle from rat, monkey and human. Arch Toxicol 1994;68:648-55.

26. Kuca K, Patocka J, Cabal J, Jun D. Reactivation of organophosphate-inhibited acetylcholinesterase by quaternary pyridinium aldoximes. Neurotox Res 2004;6:565-70.

27. Kuca K, Patocka J, Cabal J. Reactivation of organophosphate inhibited acetylcholinesterase activity by $\alpha, \omega$-bis-(4hydroxyiminomethylpyridinium)alkanes in vitro. J Appl Biomed 2003;1:207-11.

28. Mager PP. Quantitative structure-reactivity and structuretoxicity relationships of reactivators of phosphylated acetylcholinesterase. Die Pharmazie 1981;36:450-51.

29. Mager PP, Weber A. Structural bioinformatics and QSAR analysis applied to the acetylcholinesterase and bispyridinium aldoximes. Drug Des Dis 2004;18:127-50. 
30. Pang YP, Kollmeyer TM, Hong F, Lee JC, Hammond PI, Haugabouk SP, Brimijoin S. Rational design of alkylene-linked bis-pyridiniumaldoximes as improved acetylcholinesterase reactivators. Chem Biol 2003;10,491-502.
31. Kapkova P, Stiefl N, Sürig U, Engels B, Baumann K, Holzgrabe U. Synthesis, biological activity, and docking studies of new acetylcholinesterase inhibitors of the bispyridinium type. Arch Pharm 2003;336:523-40. 